\title{
Reflets
}

Revue d'intervention sociale et communautaire

\section{Stages et réalité des professionnelles et professionnels en santé et services sociaux dans un contexte francophone minoritaire à Iqaluit, au Nunavut \\ Field Practicums and the realities of health and mental health professionals working in the francophone minority context of Iqaluit, Nunavut}

\author{
Dominique Mercure, Sylvie Rivard, Roxanne Bélanger et Marie-Josée Charrier
}

Volume 24, numéro 2, automne 2018

Santé et accès aux soins de santé des communautés francophones en situation minoritaire au Canada (CFSM)

URI : https://id.erudit.org/iderudit/1053866ar

DOI : https://doi.org/10.7202/1053866ar

Aller au sommaire du numéro

Éditeur(s)

Reflets, Revue d'intervention sociale et communautaire

ISSN

1203-4576 (imprimé)

1712-8498 (numérique)

Découvrir la revue

Citer cet article

Mercure, D., Rivard, S., Bélanger, R. \& Charrier, M.-J. (2018). Stages et réalité des professionnelles et professionnels en santé et services sociaux dans un contexte francophone minoritaire à Iqaluit, au Nunavut. Reflets, 24(2), 124-153. https://doi.org/10.7202/1053866ar
Résumé de l'article

Cet article présente la démarche d'analyse et les résultats d'une recherche sur les stages et la réalité des professionnelles et professionnels en santé et services sociaux dans un contexte francophone minoritaire en région arctique. Une démarche méthodologique d'étude de cas à multiples niveaux a été choisie pour permettre de comprendre le vécu des différents acteurs et actrices de la communauté francophone vivant à Iqaluit, au Nunavut. Douze entretiens ont été effectués auprès de stagiaires et de professionnelles et professionnels oeuvrant dans les services de santé et les services sociaux pour mieux saisir leur contexte de pratique, les défis rencontrés, les compétences développées dans ce milieu et les obstacles quant à l'organisation des stages. Les résultats illustrent les perceptions de participantes et participants non autochtones sur une réalité complexe composée de nombreux défis.
Tous droits réservés (C) Reflets, Revue d’intervention sociale et communautaire, 2018
Ce document est protégé par la loi sur le droit d’auteur. L'utilisation des services d'Érudit (y compris la reproduction) est assujettie à sa politique d'utilisation que vous pouvez consulter en ligne. 


\title{
Stages et réalité des professionnelles et professionnels en santé et services sociaux dans un contexte francophone minoritaire à lqaluit, au Nunavut
}

\author{
Dominique Mercure, Ph. D. \\ Professeure à l'École de service social, Université Laurentienne \\ Sylvie Rivard, M.S.S. \\ Candidate au doctorat, professeure à l'École de service social, Université Laurentienne \\ Roxanne Bélanger, Ph. D. \\ Professeure à l'École d'orthophonie, Université Laurentienne \\ Marie-Josée Charrier \\ Maîtrise en santé interdisciplinaire, Université Laurentienne
}

\section{Résumé}

Cet article présente la démarche d'analyse et les résultats d'une recherche sur les stages et la réalité des professionnelles et professionnels en santé et services sociaux dans un contexte francophone minoritaire en région arctique. Une démarche méthodologique d'étude de cas à multiples niveaux a été choisie pour permettre de comprendre le vécu des différents acteurs et actrices de la communauté francophone vivant à Iqaluit, au Nunavut. Douze entretiens ont été effectués auprès de stagiaires et de professionnelles et professionnels œuvrant dans les services de santé et les services sociaux pour mieux saisir leur contexte de pratique, les défis rencontrés, les compétences développées dans ce milieu et les obstacles quant à l'organisation des stages. Les résultats illustrent les perceptions de participantes et participants non autochtones sur une réalité complexe composée de nombreux défis.

Mots clés : stages en région éloignée, Iqaluit (Nunavut, Canada), communauté francophone en situation minoritaire (CFSM), défis professionnels et personnels en milieu de stage, pratique professionnelle en contexte interculturel 


\section{Field Practicums and the realities of health and mental health professionals working in the francophone minority context of Iqaluit, Nunavut}

\section{Abstract}

This article presents the results of a research project on student practicums, as well as the clinical reality of professionals in health and social services living in a Francophone minority context. A multi-level case study approach was used to better understand the experiences of the various stakeholders in the Francophone community living in Iqaluit, Nunavut. Twelve interviews were conducted with students and professionals working in health and social services in order to better understand the clinical context, the challenges encountered, the skills developed in this environment, and the obstacles encountered when organizing these practicums. This article will present the perceptions of non-Indigenous participants of this complex and challenging context.

Keywords: remote placement, Iqaluit (Nunavut, Canada), Official Language Minority Community (OLMC), professional and personal obstacles during placements, professional practice in intercultural contexts

\section{Introduction}

Parmi les travaux réalisés sur les stages en région éloignée, peu de recherches se sont concentrées sur les stages francophones en contexte minoritaire et aucune dans le contexte spécifique de la communauté francophone d'Iqaluit au Nunavut. Dans cette perspective, nous visons à mieux saisir le contexte de pratique, les défis rencontrés, les compétences développées dans ce milieu et les obstacles quant à l'organisation des stages. Ainsi, nous présentons d'abord brièvement la situation sociopolitique du territoire du Nunavut et des trois langues officielles, dans laquelle s'insère cette petite communauté francophone qui se croise avec la culture inuite. Le cadre théorique choisi pour l'analyse des données réfere aux concepts entourant la compétence culturelle pour permettre de mieux comprendre les défis relevés par les stagiaires qui doivent composer avec de nouvelles réalités dans un contexte bien différent des services qu'elles connaissent. Nous décrivons ensuite les objectifs et la méthodologique utilisée pour la collecte et l'analyse des données, puis les résultats exposent les expériences des stagiaires et des professionnelles et professionnels dans ce contexte. Enfin, nous proposons une discussion en incorporant quelques conseils aux futurs stagiaires et organisatrices et organisateurs des stages. ${ }^{1}$ 


\section{Contexte de la recherche}

L'accès aux soins de santé est limité partout au Canada, particulièrement dans les communautés à densité réduite. Cette situation représente un double défi pour les francophones vivant à Iqaluit au Nunavut : avoir accès à des soins et à des services de santé, mais aussi avoir accès à ces services dans leur langue. Ces francophones, en plus de former la plus petite communauté de langue officielle en situation minoritaire (CLOSM) au Canada, habitent un territoire où l'anglais est la principale langue d'usage, bien que des politiques aient été mises en place afin de promouvoir et de préserver la langue inuite (Fédération des communautés francophones et acadienne du Canada [FCFA], 2006).

"Dans une région arctique comme le Nunavut, les stages professionnels peuvent être un moyen d'offrir des services de santé à la population francophone, tout en contribuant à long terme au recrutement de professionnelles et professionnels. "

Dans une région arctique comme le Nunavut, les stages professionnels peuvent être un moyen d'offrir des services de santé à la population francophone, tout en contribuant à long terme au recrutement de professionnelles et professionnels. Or, la mise en œuvre des stages en région à densité réduite comporte certains défis. Le présent projet interdisciplinaire a cherché à mieux comprendre les apprentissages et les défis rencontrés par les stagiaires (étudiantes à la maîtrise en orthophonie et au baccalauréat en service social), ainsi que l'impact de ces stages sur le recrutement et la rétention des professionnelles et professionnels de la santé et des services sociaux.

\section{Le Nunavut : le contexte du Qikiqtani}

Le Nunavut est un territoire canadien peu peuplé, fondé en 1999 et couvrant 1,9 million de $\mathrm{km}^{2}$. La culture est basée sur la civilisation Tulle, les chasseurs nomades voyageant par traîneau et kayak (Crago, Allen et Hough-Eyamie, 1997). Ses 31765 habitants sont dispersés dans 25 communautés isolées les unes des autres, pour lesquelles il n'existe pas de réseau routier (Statistique Canada, 2012). Les déplacements doivent s'effectuer par avion ou, l'été, par bateau. Les communautés ont adopté une technologie moderne, mais les pratiques traditionnelles demeurent fortes (Dench, et collab., 2011).

La région du Qikiqtani comprend un tiers du Nunavut, y compris l'île de Baffin, avec une population de près de 19000 habitants (Statistique Canada, 2016). Les 13 
communautés de la région varient en fonction de leur taille et de leur langue; la plupart comptent moins de 1500 résidents, dont la grande majorité se compose d'Inuits². Quant à Iqaluit, la capitale du Nunavut, elle possède des caractéristiques uniques. La ville est plus grande, avec à peu près 7700 habitants, et elle compte la plus grande proportion de non-Inuits, environ 40 \% (Statistique Canada, 2016).

La situation sociolinguistique du Nunavut est complexe. En vertu de sa Loi sur les langues officielles (2006), le Nunavut reconnaît officiellement trois langues : l'anglais, le français et la langue inuite qui comprend deux dialectes, l'inuktitut et l'inuinnaqtun

"Les Inuits, qui représentent $85 \%$ de la population du Nunavut, sont toutefois minoritaires sur le plan linguistique..."
(Leclerc, 2015). Les Inuits, qui représentent $85 \%$ de la population du Nunavut, sont toutefois minoritaires sur le plan linguistique (FCFA, 2006). L'anglais constitue la première langue officielle parlée, mais la langue inuite est encore parlée dans plusieurs foyers, puisque $54,4 \%$ de la population du Nunavut a déclaré parler le plus souvent à la maison une langue autre que l'anglais ou le français. L'inuktitut constitue le principal dialecte parlé au Nunavut, alors que l'inuinnaqtun est parlé par moins de $1 \%$ des Inuits du Nunavut, principalement dans la région de Kitikmeot, dans l'ouest du territoire (Leclerc, 2015). Or, puisque la langue inuite est en déclin à l'échelle du territoire, le gouvernement du Nunavut s'est aussi doté, en 2008, de la Loi sur la protection de la langue inuit, qui "vise la protection, la promotion et la revitalisation de la langue inuit au Nunavut " (Bureau du Commissaire aux langues du Nunavut, 2006, p. 2). Quant aux francophones du Nunavut (Nunavois), près de $90 \%$ sont aptes à parler l'anglais.

\section{Francophones du Nunavut (Nunavois)}

Selon le recensement de 2006, 465 résidents du territoire ont le français comme première langue, soit 1,6\% de la population. La majorité de ceux-ci, soit 310 individus, habitent la capitale, Iqaluit. La communauté francophone du Nunavut est non seulement très minoritaire, mais elle se distingue aussi des autres communautés minoritaires du pays, où les francophones qui y vivent sont intégrés et forment une communauté depuis plusieurs

"Bien que les francophones du Nunavut revendiquent des services offerts dans leur langue, leur intégration à la société nunavoise demeure difficile..." générations. En fait, huit francophones sur dix qui habitent au Nunavut sont nés hors du territoire, et leur durée moyenne de séjour est de deux à trois ans. Bien que les francophones du Nunavut revendiquent des 
services offerts dans leur langue, leur intégration à la société nunavoise demeure difficile, alors que l'anglais et l'inuktitut continuent de primer. Malgré le fait que ces individus arrivent de toutes les provinces canadiennes, la grande majorité provient du Québec. Ces francophones sont largement des adultes, puisque les moins de 20 ans ne comptent que pour $19 \%$ de la population (FCFA, 2006).

Un francophone sur deux travaille en administration publique, en éducation, en soins de santé ou en service social, tandis qu'environ $11 \%$ œuvrent dans le domaine du transport et de l'entreposage et $7 \%$ en construction et en commerce de gros et de détail. Enfin, environ 30 individus francophones travaillent à leur propre compte, représentant $8,7 \%$ de la main-d'œuvre francophone (FCFA, 2006).

\section{Accès aux services de santé et de santé mentale en français}

De manière générale, il est maintenant bien documenté que les francophones qui ne peuvent pas communiquer adéquatement (langue des services, niveau d'alphabétisation) n'auront pas accès à la même qualité de soins (Bouchard, et collab., 2006). Or, des études plus explicites démontrent que, dans le domaine de la santé, la langue de communication a un impact sur la qualité, l'efficacité et la sécurité des services (Bouchard, et collab., 2009; Bouchard, et collab., 2006; Forgues et Landry, 2014). Lorsqu'il y a présence de barrières linguistiques, celles-ci peuvent mener à une sous-utilisation des services par les groupes minoritaires (Riddick, 1998).

Par rapport aux services offerts dans leur langue, les Nunavois ont dû faire plusieurs revendications pour avoir accès à une meilleure offre de services en français. Or, le contexte de ce territoire est complexe, influencé par les politiques linguistiques territoriales qui accordent à l'inuktitut un statut officiel au même titre que le français et l'anglais, et qui visent aussi à protéger, préserver et transmettre la langue. Ainsi, les demandes et les

les demandes et les revendications de la communauté francophone doivent tenir compte des exigences de la Loi sur la protection de la langue inuit et des politiques mises en place par le gouvernement du Nunavut. » revendications de la communauté francophone doivent tenir compte des exigences de la Loi sur la protection de la langue inuit et des politiques mises en place par le gouvernement du Nunavut. Par conséquent, l'intégration des francophones au Nunavummiut ${ }^{3}$ demeure difficile dans ce contexte particulier. 


\section{Stages professionnels}

Les programmes universitaires ont la responsabilité sociale d'offrir aux étudiantes et étudiants des occasions d'acquérir les aptitudes et les capacités nécessaires afin de répondre aux normes de compétence de la profession et à l'entrée en pratique. En Ontario, les étudiantes et étudiants dans divers programmes de formation professionnelle doivent compléter des formations pratiques (par exemple : en orthophonie, 350 heures cliniques dans des milieux variés, et en travail social, 700 heures). Parmi les compétences essentielles exigées par les ordres professionnels respectifs, on trouve la sensibilité culturelle et linguistique, la compétence culturelle étant un attribut important pour toute discipline qui souhaite travailler efficacement auprès de personnes provenant d'origines diverses (Jones, Neubrander et Huff, 2012).

\section{"Dans des régions à densité réduite comme les territoires du Grand Nord canadien, les expériences de stages permettent d'offrir des services de santé et services sociaux dans ces régions sous- desservies."}

Dans des régions à densité réduite comme les territoires du Grand Nord canadien, les expériences de stages permettent d'offrir des services de santé et services sociaux dans ces régions sous-desservies. De plus, puisque ces régions font face à des obstacles quant au recrutement de professionnelles et professionnels francophones de la santé et de la santé mentale, les stages cliniques peuvent aussi y contribuer à long terme (Consortium national de formation en santé, 2015). Pour pallier cette lacune, ainsi que pour fournir aux étudiantes et étudiants l'occasion de parfaire leur sensibilité culturelle et linguistique, plusieurs programmes universitaires au Canada cherchent à créer des milieux de formation clinique dans ces régions.

\section{Stages interculturels}

"... une fois immergés dans l'autre culture, les étudiantes et étudiants commencent à examiner leurs propres visions du monde, à développer une perspective plus globale..."
L'influence de l'immersion culturelle des stagiaires sur le développement de leurs compétences essentielles est l'objet de plusieurs recherches, entre autres, en sciences infirmières où l'on rapporte comment les étudiantes et étudiants peuvent améliorer leur capacité à fournir des
vers milieux culturels (Button, et collab., 2005; soins efficaces aux individus issus de divers milieux culturels (Button, et collab., 2005; Callister et Cox, 2006). Ces études montrent que les expériences d'immersion culturelle 
favorisent la croissance personnelle et professionnelle des étudiantes et étudiants (Evanson et Zust, 2004). De plus, une fois immergés dans l'autre culture, les étudiantes et étudiants commencent à examiner leurs propres visions du monde, à développer une perspective plus globale concernant les systèmes de soins de santé en dehors de leur région (Walsh et DeJoseph, 2003).

\section{Cadre théorique}

Pour bien expliquer l'importance de la culture dans les déterminants de la santé et en tenir compte dans l'offre de services, Bélanger (2002) explique comment des personnes de différentes cultures n'accordent pas le même sens à un même problème. Ainsi, le stage interculturel implique une prise de conscience de la diversité culturelle et l'adoption de pratiques tenant compte de cette diversité. Le travail d'adaptation au contexte devient un apport majeur sur le plan de la formation. Le stage interculturel permet ainsi de saisir le fonctionnement social dans un autre contexte que le sien; il appelle aussi à une vision critique des systèmes, à une adaptation au milieu, à une nécessité de collaboration pour pouvoir composer avec les différents obstacles rencontrés et à un ancrage des valeurs professionnelles (Mercure, Ba et Turcotte, 2010).

Cohen-Emerique (1984) décrit le phénomène du choc culturel interférant dans ce type de stage. Elle définit cette expérience comme l'état de tension survenant lorsque des personnes de différentes cultures découvrent et explorent l'étrangeté de leurs attitudes et comportements. Du dépaysement amusant au départ naît un nouveau sentiment de malaise et d'incompréhension; l'individu façonne l'interprétation des situations au moyen des repères de sa propre culture et de là émergent des réactions non adaptées aux situations nouvelles (Cohen-Emerique, 1984). La décentration devient alors préalable à la découverte du cadre de référence de l'autre culture, une ouverture pour aller à la rencontre de l'autre, prenant conscience de soi afin de "mieux cerner ses cadres de référence, d'en prendre conscience en tant qu'individu porteur d'une culture et de sous-cultures » (Cohen-Emerique, 2011, p.175). Ce processus apporte un éclairage sur les distorsions possibles dans la compréhension de l'autre, suscitées par le choc culturel, et il permet de repérer et d'analyser les zones sensibles qui sont activées par l'interaction avec l'étranger (Cohen-Emerique, 2011). Il s'agit d'un exercice essentiel pour les stagiaires qui doivent s'adapter aux réalités présentes dans le contexte des services à offrir au Nunavut; ces stagiaires doivent donc être préparés à cette réflexion préalablement à leur séjour de stage. 


\section{Compétence culturelle : un processus}

Le modèle de Campinha-Bacote (2002) repose sur le principe que la compétence culturelle est un processus et non une finalité, où le professionnel ou la professionnelle de la santé travaille de façon efficace dans le contexte culturel de l'individu, de la famille "La compétence culturelle fait appel à une intégration de la conscience culturelle, du savoir culturel, des habiletés culturelles, des rencontres culturelles et du désir culturel. " ou de la communauté. Il s'agit d'un processus qui fait appel à une intégration de la conscience culturelle, du savoir culturel, des habiletés culturelles, des rencontres culturelles et du désir culturel. Dans ce modèle, la notion de compassion (caring) doit dépasser le respect des valeurs, croyances et pratiques; elle implique la motivation profonde à s'engager dans le processus de la compétence culturelle et à offrir des soins qui répondent aux besoins culturels. Cet engagement suggère la volonté d'être flexible et d'accepter les différences, de s'appuyer sur les similitudes ou d'être disposé à apprendre en voyant l'autre comme une source d'information culturelle. Ce genre d'apprentissage est un processus continu qui a déjà été défini comme étant l'humilité culturelle (Trevalon et Murray-Garcia, 1998, cité dans Campinha-Bacote).

Blanchet Garneau et Pepin (2012) font une recension des écrits liés à un nouveau concept rattaché à la compétence culturelle, celui de la sécurité culturelle, qui implique un partenariat égalitaire, une participation active des personnes ainsi que la protection de l'identité culturelle et du bien-être. Les forces et les capacités des deux parties sont ainsi prises en compte pour déterminer les stratégies et les priorités de soins (Blanchet Garneau et Pepin, 2012). La sécurité culturelle suppose un engagement sincère de chacun sur les plans cognitif, affectif et comportemental, et ce, à toutes les étapes de l'intervention.

Cette approche veut assurer la protection de l'identité culturelle et du bien-être et apporte une dimension morale à la sécurité culturelle en faisant état d'un souci de justice sociale. Elle vise à réduire le potentiel d'exploitation ou d'une approche stéréotypée de la culture (Blanchet Garneau et Pepin, 2012). Ces dimensions sont d'autant plus pertinentes pour pouvoir offrir des services culturellement sensibles dans le contexte du Nunavut dans lequel s'insère ce projet de recherche. Cette compréhension permet ainsi une reconnaissance de la légitimité des diversités et des impacts qu'une intervention peut avoir sur les autres. Ainsi, il ne s'agit pas de posséder des connaissances spécifiques au sujet d'une culture, mais plutôt d'avoir des connaissances à propos des inégalités et de comprendre comment le contexte influence l'état de santé des individus et des communautés, tout en 
cherchant à identifier les causes historiques, sociales, culturelles, politiques et économiques des inégalités de santé et de soins (Blanchet Garneau et Pepin, 2012).

Finalement, trois conséquences émergent de la sécurité culturelle : l'émancipation, l'efficacité et la qualité des soins et, enfin, l'égalité et la justice sociale. Cependant, puisqu'à ce jour, il n'existe pas de données empiriques pour appuyer les effets réels de la sécurité culturelle, ces principes deviennent une manière d'aborder la diversité avec une sensibilité accrue et un regard critique sur les approches préconisées.

\section{Objectifs du projet de recherche}

Bien que l'équipe de recherche soit sensible au contexte sociohistorique de la colonisation et à l'oppression linguistique des communautés inuites du Nunavut, l'objectif du projet demeure de mieux comprendre les besoins de la communauté francophone nunavoise. Ainsi, les données présentées dans cet article n'ont pas pour but de faire un tour exhaustif des multiples dimensions influençant le contexte des services à offrir au Nunavut, mais bien de décrire d'un point de vue non autochtone le vécu des stagiaires et des professionnelles et professionnels impliqués dans l'offre des services en santé.

Ce projet interdisciplinaire, créé par trois chercheures de deux écoles impliquées dans ces stages (orthophonie, service social), a été développé en collaboration avec le Réseau

"Cette démarche collaborative visait à mieux comprendre les apprentissages et les défis rencontrés par les stagiaires, ainsi que l'impact de ces stages sur le recrutement et la rétention des professionnelles et professionnels de la santé et des services sociaux." de santé en français au Nunavut (RÉSEFAN). Cette démarche collaborative visait à mieux comprendre les apprentissages et les défis rencontrés par les stagiaires, ainsi que l'impact de ces stages sur le recrutement et la rétention des professionnelles et professionnels de la santé et des services sociaux. Les objectifs spécifiques étaient les suivants :

1. Mieux comprendre le portrait démographique de la population francophone d'Iqaluit et analyser les besoins liés aux professions œuvrant dans les services de santé et services sociaux.

2. Décrire l'impact (à court et à long terme) de l'implication de stagiaires dans une communauté à densité réduite.

3. Analyser les apprentissages professionnels des stagiaires engagés dans un stage à Iqaluit. 
4. Comprendre le contexte de recrutement et de rétention des professionnelles et professionnels de la santé et des services sociaux, soit au niveau des politiques organisationnelles et gouvernementales, ainsi que les facteurs structuraux agissant sur ce contexte.

\section{Méthodologie : une étude de cas à multiples niveaux}

Une méthodologie de recherche qualitative s'échelonnant dans le temps a été privilégiée en raison des caractéristiques particulières de la communauté francophone vivant à Iqaluit, de la problématique qui découle du contact des langues et du contexte de colonisation qui a marqué l'histoire récente du peuple inuit, des difficultés que posent le recrutement et la rétention des professionnelles et professionnels de la santé à Iqaluit et des défis inhérents aux stages de formation professionnelle en région nordique. Il importait de pouvoir rendre compte non seulement des particularités du milieu, mais aussi d'un projet en mouvance et des facteurs gouvernementaux et structuraux qui influencent la problématique en question. Ainsi, nous avons choisi une méthodologie de recherche développée par Caronna (2010), qui définit l'étude de cas à multiples niveaux, incluant une analyse du microsystème, du mésosystème et du macrosystème. Cette méthodologie puise aux principes traditionnels de l'étude de cas et de l'ethnographie organisationnelle dans une perspective systémique. De plus, notre démarche a été appuyée par des principes collaboratifs et itératifs d'une recherche-action, menée par une équipe interdisciplinaire engagée dans un processus longitudinal. Ici, nous expliquons tout d'abord ces deux méthodologies de recherche, leur croisement au sein de ce projet en lien avec les concepts définis dans le cadre théorique. Par la suite, nous abordons l'opérationnalisation du processus de collecte des données afin d'expliquer le traitement et l'analyse des données.

Caronna (2010) identifie la difficulté de dégager, par le biais des méthodes de recherche traditionnelles, les liens entre les politiques touchant la santé dans son ensemble, les structures organisationnelles des services, les expériences et les droits des récipiendaires de services de santé et de services sociaux, l'état de santé des individus et la culture et, finalement, les valeurs et croyances sociétales. Ainsi, elle propose l'utilisation de l'étude de cas à multiples niveaux afin de rendre compte de la complexité de l'intersection de ces paliers, soit le microsystème (individuel), le mésosystème (organisationnel) et le macrosystème (environnemental) (Caronna, 2010). L'étude de cas à multiples niveaux vise à dégager l'analyse d'une situation donnée ou en processus, tout en explorant l'influence des contextes sociaux, politiques et structurels sur la situation ou l'expérience 
donnée (Caronna, 2010). D’une part, cette méthodologie s'intéresse à des phénomènes, particulièrement lorsque les frontières entre le phénomène et le contexte sont parfois floues, et elle utilise de multiples sources de données, telles que des entretiens, des documents et de l'observation sur le terrain. D'autre part, l'ethnographie institutionnelle implique des observations appuyées par les notes de terrain tenues par la chercheure ou le chercheur et les entretiens avec des actrices et acteurs clés qui forment le corpus des données de la recherche.

Dans la présente recherche, découlant des besoins exprimés par la communauté francophone d'Iqaluit, nous utilisons aussi des principes de recherche-action. Dolbec (2003) indique que le modèle de recherche-action est centré sur les communautés pour une meilleure compréhension des besoins réels du terrain et des pratiques de l'action. Ainsi, dans une perspective de collaboration avec la communauté francophone d'Iqaluit, diverses rencontres ont eu lieu préalablement et tout au long de la collecte de données, en vue de bâtir ces collaborations avec les divers partenaires de la communauté francophone d'Iqaluit et de cibler les thématiques à exploiter.

- Visant à comprendre l'implication de stagiaires dans les services de santé et services sociaux en français à Iqaluit et l'effet sur le recrutement et la rétention de la main-d'œuvre dans ce milieu, l'étude de cas à multiples niveaux cible :

- Au niveau du microsystème (individuel) : l'expérience des stagiaires, les apprentissages, les expériences liées à la minorité linguistique, les effets de l'isolement d'un milieu arctique et du contexte interculturel sur le vécu du stagiaire;

- Au niveau du mésosystème (organisationnel) : les besoins de services en santé et services sociaux à Iqaluit, les enjeux vécus par la communauté francophone, tels qu'exprimés par les professionnelles et professionnels travaillant dans les services de santé et services sociaux;

- Au niveau du macrosystème (environnemental) : l'impact des stages sur la communauté d'accueil (à court terme et à plus long terme), la perspective des décideurs gouvernementaux, l'analyse documentaire de l'évolution des politiques touchant les services en français au Nunavut, le recrutement et la rétention des professionnelles et professionnels francophones. 


\section{Opérationnalisation : collecte des données}

La mise en œuvre de la collecte des données et de l'analyse de ces dernières s'est effectuée dans une séquence de trois volets distincts et dans un processus itératif et inductif où les données recueillies à une étape ont servi d'éclairage aux suivantes (Caronna, 2010).

Un premier canevas d'entretien a été développé au terme des rencontres avec les partenaires de recherche de la communauté francophone d'Iqaluit et d'une recension des écrits sur l'objet de la recherche. Dans la première séquence de collecte de données (microsystème), nous avons recruté des étudiantes de programmes professionnels en santé et en services sociaux d'institutions postsecondaires impliquées dans un stage professionnel à Iqaluit dans des milieux variés, et en interaction avec des clientèles francophones, inuites et anglophones, mais dont certains étaient principalement francophones. Quatre étudiantes en orthophonie (à la maîtrise) et deux étudiantes en service social (à la technique et au baccalauréat) ont participé à des entretiens semi-dirigés en amont et en aval de leur expérience de stage sur les aspects suivants : la formation universitaire et leurs intérêts professionnels, le stage interculturel et les défis anticipés, le contexte de minorité francophone et en région éloignée, les activités d'apprentissage et le contexte de travail, et le bilan de leur expérience en lien avec leur devenir professionnel. Tous les entretiens ont été enregistrés, puis transcrits afin de présenter l'intégralité de leurs propos. Les informations ont aussi été anonymisées en vue de protéger l'identité des participantes et participants, faciles à reconnaître dans un petit milieu comme Iqaluit. Des catégories thématiques ont été utilisées afin d'effectuer une première codification et une analyse préliminaire des données. Nous nous sommes attardées à repérer et à codifier les thèmes portant sur leurs expériences personnelles (émotions et perceptions) et leurs impressions de la communauté et de ses besoins, et des apprentissages professionnels liés à leur expérience du stage.

Ce processus a ainsi permis de développer un deuxième canevas de questions ciblant le volet du niveau mésosystème de la recherche. Nous avons réalisé des entretiens avec six professionnelles et professionnels œuvrant dans la communauté des services de santé et des services sociaux afin d'explorer leur choix de travailler à Iqaluit, les défis rencontrés dans leur pratique et leurs perceptions des besoins de la communauté francophone d'Iqaluit. Ce recrutement s'est effectué au sein des participantes et participants ayant été identifiés par les partenaires communautaires et parce qu'elles et ils sont des acteurs clés dans l'offre des services de santé et services sociaux en français à Iqaluit. Les entretiens ont alors été codifiés à partir des thèmes ayant émergé du premier volet, puis mis en lien avec les écrits 
scientifiques et le contexte sociopolitique actuel, répertoriés lors de la mise en place de la problématique et du cadre théorique. Par la suite, les résultats ont été partagés et discutés avec les partenaires communautaires afin d'amorcer la démarche d'un troisième volet.

Pour le dernier volet ciblant le macrosystème, nous avons rencontré des personnes issues de la structure politique, gouvernementale et décisionnelle des services de santé et des services sociaux du Nunavut. Dans celui-ci, nous avons suivi les mêmes boucles d'induction dans la démarche de construction du canevas d'entretien, pour le recrutement des participantes et participants, pour les entretiens et pour la codification des verbatims et l'analyse des données. Au total, seize entretiens ont été effectués pour ce volet des décideurs.

Nous avons choisi de procéder à des analyses par processus inductif, dans une construction des thèmes et des catégories qui émergent des entretiens. Pour chaque volet de la codification thématique (Saldaña, 2013), un va-et-vient de comparaison entre les participantes et participants (comparaison horizontale de cas à cas) (Miles, Huberman et Saldaña, 2014) a permis de dégager des similarités et des différences. Enfin, une relecture des écrits scientifiques à chaque étape a aussi permis de contextualiser la classification des thèmes émergents, en lien avec les objectifs de recherche et les concepts exploités.

\section{Résultats}

Dans le cadre de cet article, nous ne présentons que les résultats de deux volets, soit ceux du niveau micro (les résultats des entretiens réalisés avec les stagiaires) et du niveau méso (les résultats des entretiens réalisés avec des professionnelles et professionnels de la santé et des services sociaux d'Iqaluit). Les résultats issus du troisième volet (niveau macro) ainsi que les analyses entre les divers niveaux feront l'objet d'un article ultérieur.

\section{Premier volet : Expérience des stagiaires}

À la lumière des entretiens effectués avec les stagiaires, l'expérience de stage à Iqaluit est essentiellement décrite comme riche en apprentissages. Les analyses permettent de discuter de quatre thèmes principaux :

1) les défis de la communication interculturelle,

2) la capacité d'adaptation,

3) le professionnalisme, et

4) l'expérience francophone. 


\section{Thème 1 : Défis de la communication interculturelle}

La communication en contexte interculturel est l'un des principaux défis des stagiaires. En effet, certaines des personnes rencontrées parlent uniquement l'inuktitut et, si elles peuvent parler l'anglais, il s'agit de leur langue seconde. C'est une situation qui peut affecter la $\mathrm{qu}^{\mathrm{TM}}$ alité des services offerts, car les stagiaires doivent s'assurer de transmettre l'information et les recommandations de façon claire et précise tout en s'assurant que la personne a bien compris.

Afin de bien communiquer avec les personnes qui utilisent les services, les stagiaires peuvent utiliser les services d'interprètes. Or, le travail avec des interprètes inuits ne facilite pas toujours le partage d'information et soulève certaines questions déontologiques. Par exemple, puisque les interprètes sont des membres issus de la communauté inuite, ils peuvent en plus et bien souvent être un membre de la famille de la personne qui utilise des services. Dans de telles situations, il devient parfois difficile d'assurer la confidentialité selon les normes professionnelles. De plus, comme il peut être impossible de vérifier l'exactitude de l'information transmise par l'interprète au bénéficiaire des services, l'obtention d'un consentement éclairé et la bonne compréhension des enjeux liés aux soins et au plan de traitement proposés peuvent devenir problématiques.

«Les professionnels viennent du Sud, donc ils parlent l'anglais, le français peutêtre, mais ils ne parlent pas l'inuit [inuktitut]. Pour communiquer et offrir l'information en inuit [inuktitut], ils embauchent des personnes pour traduire les documents, mais c'est une traduction. Il y a aussi la situation de l'Inuit qui connaît l'anglais et l'inuit [inuktitut]; donc il peut traduire, mais c'est limité, surtout pour un document scientifique... c'est pas toujours évident. Donc c'était vraiment difficile d'essayer de fournir des services dans leur langue dominante. Tu regardes les documents que tu donnes aux parents, mais tu ne sais pas si ça dit la bonne chose, car tu ne sais pas exactement ce qu'il traduit. En plus, ils ne comprennent pas facilement les services, puis, là, ils ne comprennent pas nécessairement ce que tu leur présentes. Des fois, il y a un manque de compréhension à plusieurs niveaux. "

Pour les stagiaires, l'utilisation de la communication non verbale peut aider à communiquer en situation interculturelle. Toutefois, certains codes culturels pourraient être mal interprétés si les stagiaires ne connaissent pas suffisamment les repères nécessaires pour apprendre à reconnaitre ce non-verbal. Plus encore, les stagiaires doivent pouvoir développer une pensée critique afin d'évaluer et d'analyser les résultats en fonction des 
référents culturels des personnes qui utilisent des services, plutôt que ceux établis dans leur propre culture. Pour cette raison, les stagiaires trouvent parfois difficile de faire la distinction entre une problématique réelle en santé ou en santé mentale et une différence culturelle, d'autant plus que leurs connaissances de l'inuktitut et de la culture inuite sont rudimentaires, comme nous l'explique cette participante :

"J'avais lu que, dans leur culture, les enfants apprennent en écoutant et en observant, alors les parents ne se sentent pas le besoin de leur parler, car l'enfant va apprendre en situation avec l'environnement... alors que nous, c'est important de l'exposer au vocabulaire pour bâtir le langage. C'est tellement différent. Les étapes de développement ne correspondent pas à nos normes. »

De plus, les stagiaires constatent que de bonnes habiletés de communication sont nécessaires afin d'établir une relation de confiance avec la population inuite qui présente une certaine méfiance à l'égard des Qallunaat (personnes blanches), et ce, en raison des effets de la colonisation. À certains moments, les stagiaires font face à des réticences de la part des personnes utilisant des services qui, en raison de leurs croyances et de leur culture, n'ont pas la même perspective quant à la nécessité des traitements ou la manière de gérer leur situation médicale. Elles soulignent alors toute l'importance de transmettre l'information de façon claire et de répondre aux questions de façon respectueuse. Finalement, pour les stagiaires, c'est aussi une occasion de se présenter dans leur rôle

"À Iqaluit, l'expérience interculturelle est une composante importante du stage qui amène les stagiaires à développer cette sensibilité culturelle qui leur permet de comprendre la perception des familles, de respecter leurs valeurs et de faire en sorte que la perspective du bénéficiaire soit intégrée aux services qui répondront à ses besoins en tenant compte des différences culturelles." professionnel en expliquant aux familles la façon dont elles peuvent les aider.

À Iqaluit, l'expérience interculturelle est une composante importante du stage qui amène les stagiaires à développer cette sensibilité culturelle qui leur permet de comprendre la perception des familles, de respecter leurs valeurs et de faire en sorte que la perspective du bénéficiaire soit intégrée aux services qui répondront à ses besoins en tenant compte des différences culturelles.

"Tu essaies d'apporter ta contribution avec une ouverture d'esprit, mais tu dois demeurer humble, car ce n'est pas ta culture. »

"Les traditions sont très riches et très intéressantes : leur lien avec la Terre, leur nutrition et le bris créé par la vie moderne où tout coûte cher [...] c'est 
complexe à comprendre, puis tu rencontres de nombreux problèmes liés à l'alcoolisme foetal, le viol et l'inceste..."

\section{Thème 2 : Capacité d'adaptation}

Parmi les raisons pour lesquelles les participantes à cette recherche ont choisi de faire un stage à Iqaluit, elles mentionnent le désir de vivre une expérience unique et différente, mais, surtout, l'importance de l'occasion qui leur est offerte de découvrir une toute autre culture. Les stagiaires décrivent leur arrivée à Iqaluit comme un "choc ». Elles se disent dépassées par l'ampleur de la tâche professionnelle à accomplir. En effet, elles soulignent les longues listes d'attente pour l'obtention de services, les besoins multiples et complexes des personnes utilisant les services, et que cela dépasse largement la capacité des services offerts. Par exemple, il n'y a qu'un seul orthophoniste qui dessert Iqaluit. Les stagiaires en orthophonie doivent donc intervenir avec une clientèle de tous les âges présentant des pathologies très variées. Elles doivent s'adapter rapidement en faisant appel à un ensemble de connaissances et de notions vues en salle de classe. Pour elles, il s'agit d'une expérience exigeante et différente des autres stages qu'elles ont effectués et qui sont généralement plus centrés sur des groupes particuliers. Par conséquent, les stagiaires doivent être polyvalentes, faire preuve d'initiative et travailler de façon autonome, comme l'exprime cette participante :

«Tu dois être forte. C'est vraiment émotif d'entendre toutes ces histoires et de ne pas toujours savoir quoi faire. Il faut reconnaître qu'on va vivre des chocs. J'ai eu à visiter un quartier pauvre, un appartement où quinze personnes vivent ensemble, et les vitres brisées avec ce climat. Ça m'a choquée de voir ces injustices. J'ai vu des enfants qui ne mangeaient pas pendant quelques journées et des blessures sur leurs visages. »

Les stagiaires doivent aussi ajuster leurs pratiques afin de répondre aux besoins des personnes qu'elles accompagnent dans une perspective qui demande de faire preuve de flexibilité. Par ailleurs, l'environnement de travail et les horaires plus souples incluent aussi une politique de " porte ouverte " pour les rendez-vous afin de répondre aux besoins de la communauté inuite Nunavummiut, qui est parfois difficile à rejoindre si l'on fixe des moments de rencontre prédéterminés. Agir avec flexibilité permet donc de revoir les priorités en fonction des habitudes de vie particulières des personnes qui utilisent des services, mais aussi de composer avec un modèle de prestation de services qui n'est pas uniquement axé sur les besoins de la personne. En effet, les stagiaires doivent parfois 
composer avec toute la famille qui se présente au rendez-vous :

«Une maman se présente avec quatre enfants et les amène dans la salle. Tu dois jouer avec tous les enfants, mais tu essaies d'intervenir avec l'enfant en besoin. Les autres enfants ont peut-être des problèmes eux aussi, mais ils sont sur la liste d'attente. Tu essaies de tout coordonner. »

" Je suis quelqu'un qui aime la structure et les horaires; je pense que je suis maintenant meilleure à être plus flexible avec cette expérience où j’ai appris : Just go with the flow [...] mais, au début, ça me stressait beaucoup. "

"... les stagiaires doivent apprendre à reconnaître les éléments culturels afin de bien cibler les besoins des personnes qui utilisent les services. Par ailleurs, les stagiaires rencontrées rapportent qu'elles doivent faire preuve de créativité afin de pallier le manque de matériel auquel elles sont habituées et de concevoir des activités qui répondent aux besoins du client."
Ainsi, les stagiaires doivent apprendre à reconnaître les éléments culturels afin de bien cibler les besoins des personnes qui utilisent les services. Par ailleurs, les stagiaires rencontrées rapportent qu'elles doivent faire preuve de créativité afin de pallier le manque de matériel auquel elles sont habituées et de concevoir des activités qui répondent aux besoins du client. Par exemple, en orthophonie, l'évaluation et l'intervention au niveau de la parole et du langage ont longtemps été difficiles en raison de la carence d'outils. Certes, plusieurs outils et programmes ont été développés en français au Québec, et il serait souhaitable d'avoir accès à ces outils ailleurs au Canada. Toutefois, il existe d'importantes différences liées au contexte linguistique spécifique à la situation minoritaire.

Certaines stagiaires abordent les stratégies d'adaptation personnelle qu'elles ont dû déployer pour composer avec l'isolement et s'impliquer dans la communauté durant la période de leur stage. Plusieurs notent que la connaissance de soi et de ses limites permet une réflexion importante pour leur future carrière. Par ailleurs, le soutien offert par la supervision et par le partenaire d'accueil est aussi déterminant pour assurer la réussite des objectifs professionnels durant le stage, comme le précise cette participante :

" Il fait super froid, parfois moins de 50 degrés et il y a peu de soleil. On avait des blizzards de deux jours, on était donc enfermées à la maison pour deux jours. J'ai appris à passer le temps en parlant avec mes colocataires, à m'exprimer et écouter ce qu'ils vivent ici." 


\section{Thème 3 : Professionnalisme}

Les résultats montrent que l'expérience de stage à Iqaluit a permis aux stagiaires de se familiariser avec le concept de sécurité culturelle (Blanchet Garneau et Pepin, 2012) et de poser un regard différent sur leur rôle professionnel. Les stagiaires sont aussi touchées par l'ampleur des problématiques sociales et par les conditions de vie difficiles des Inuits, liées entre autres aux effets de la colonisation. Elles reconnaissent l'importance de développer une ouverture d'esprit afin de comprendre les différences, sans porter de jugement, même dans l'ébranlement de leurs valeurs personnelles. Elles se sentent souvent impuissantes face aux situations rencontrées. L'immersion dans cet environnement amène les stagiaires à reconnaître et à apprécier les ressources et le soutien dont elles bénéficient dans leur milieu d'origine, en plus d'éveiller un sentiment d'humilité. Selon les observations des participantes, certaines personnes arrivent à Iqaluit avec tous leurs savoirs et l'idée de tout changer, alors qu'il faut plutôt viser l'autodétermination des personnes. Ainsi, il est important de connaître l'histoire des Inuits et de s'intéresser à leur culture, tout comme de prendre conscience de l'injustice et des iniquités de la distribution de ressources quant aux conditions de vie.

Les stagiaires sont aussi conscientes que leurs outils et méthodes d'intervention ont été conçus dans la culture dominante. Comme l'indique cette participante, les stagiaires doivent s'adapter à la culture inuite, puisque l'orthophonie est une jeune profession, méconnue au Nunavut, et qu'il faut tenir compte du fait que plusieurs personnes qui utilisent les services ne comprennent pas ce que sont les services d'orthophonie et ne savent pas pourquoi elles doivent voir un orthophoniste :

«On a dû faire beaucoup d'évaluations informelles, car les tests ne conviennent pas à la culture inuite; on ne pouvait pas utiliser les rangs centiles, donc nos interprétations étaient basées sur nos observations et les entrevues faites avec le point de vue des parents. "

Même si elles désirent aider et faire une différence dans la vie des gens, les stagiaires rencontrées constatent les limites de leur profession et de la relation d'aide en contexte inuit. Les ressources et le temps sont limités, et elles ne peuvent aider tout le monde. Malgré tout, elles se disent beaucoup plus confiantes sur le plan professionnel à la suite de leur stage à Iqaluit. Le fait d'avoir à gérer les dossiers des personnes qu'elles accompagnent et de répondre à un besoin en étant valorisées a contribué à accroître cette confiance. C'est d'ailleurs ce qu'évoque cette stagiaire : 
"J'ai développé des habiletés pour m’adapter aux situations. Être flexible, avoir l'esprit ouvert, l'empathie, l'humilité, avoir une pensée critique... Et à force de faire du problem solving [résolution de problème], j'ai développé de l'autonomie puis de la confiance en mes compétences. »

Plusieurs stagiaires relatent avoir développé des compétences précises en counseling et en enseignement. Certaines situations ont permis d'approfondir les habiletés en relation d'aide, par exemple l'annonce de mauvaises nouvelles. Pour les stagiaires, l'enseignement ajoute une dimension importante à la relation thérapeutique parce qu'il permet à la professionnelle ou au professionnel « d'aider la personne à s'aider », c'est-à-dire en misant sur ce qu'elle sait, ce qu'elle doit faire et comment elle peut le faire.

"Le fait de pouvoir compter sur le soutien d'une équipe multidisciplinaire est important en milieu communautaire en région éloignée, tout particulièrement à Iqaluit. »

Le fait de pouvoir compter sur le soutien d'une équipe multidisciplinaire est important en milieu communautaire en région éloignée, tout particulièrement à Iqaluit. La professionnelle ou le professionnel ne peut pas tout faire et doit pouvoir diriger le bénéficiaire vers les bonnes ressources. En ce sens, comme l'indique une stagiaire, la collaboration entre professionnelles et professionnels est primordiale :

«Avec les ressources limitées ici, la collaboration entre professionnels de la santé constitue le meilleur outil d'un thérapeute.»

En contrepartie, une stagiaire constate aussi que le fait de travailler dans une petite communauté, comme celle d'Iqaluit où tout le monde se connaît, apporte aussi des défis reliés à la confidentialité. Lorsqu'on est la seule ou le seul spécialiste de sa discipline à Iqaluit, certaines des personnes qui utilisent des services peuvent aussi être des amis ou collègues de travail. Il est alors nécessaire de faire preuve de professionnalisme, même si la frontière est parfois mince entre le rôle professionnel et la relation personnelle.

\section{Thème 4 : Expérience francophone}

Faire un stage à Iqaluit, c'est aussi l'occasion de côtoyer d'autres francophones qui vivent en situation linguistique minoritaire et d'offrir des services professionnels en français. Les stagiaires constatent la vitalité de cette communauté dynamique qui offre diverses ressources et occasions de participer à des activités francophones variées, comme l'explique cette participante : 
"L'implication dans les activités de la communauté francophone m’a permis de ne pas me refermer sur moi, malgré le sentiment d'isolement. »

Le fait de voir qu'il est possible de vivre sa francophonie, malgré la petite taille de la communauté, contribue au renforcement de la confiance identitaire, surtout chez l'une des stagiaires dont la langue maternelle est le français, mais qui, en raison du statut minoritaire de la langue française dans sa communauté d'origine, se sent beaucoup plus à l'aise de parler l'anglais. C'est d'ailleurs cette expérience positive qui, selon elle, lui a donné la confiance nécessaire pour accepter un poste dans un organisme francophone dès la fin de sa scolarisation, en plus de renforcer la valeur qu'elle accorde à la langue française :

"...je n'avais pas réalisé que cette expérience mavait poussée vers la carrière que je viens de choisir dans un poste francophone, parce qu'on mia donné confiance dans ma capacité de fonctionner en français. " "
"Je n'avais pas réalisé l'impact du stage, car j’ai surtout parlé des défis; je n'avais pas réalisé que cette expérience m'avait poussée vers la carrière que je viens de choisir dans un poste francophone, parce qu'on m'a donné confiance dans ma capacité de fonctionner en français. »

Certaines des stagiaires projettent aussi de vouloir s'investir dans des communautés en région éloignée, car elles ont beaucoup apprécié l'expérience de vivre dans ce coin de pays en pleine nature et la solidarité professionnelle et linguistique observée à Iqaluit.

\section{Deuxième volet : Contexte de pratique des professionnelles et professionnels}

Afin de mieux comprendre l'expérience des stagiaires dans une communauté à densité réduite, nous avons analysé des segments des données du volet professionnel pour approfondir les thèmes rapportés par les stagiaires lors des entretiens.

Bien que la région d'Iqaluit offre de nombreux emplois stimulants et des occasions de relever des défis, les professionnelles et professionnels de la santé qui sont en poste nous racontent être surchargés parce que les besoins sont grands, mais aussi parce que la clientèle contourne les méthodes typiques de prise de rendez-vous en s'adressant directement aux professionnelles et professionnels, parfois en dehors des heures normales de travail. Malgré la lourdeur des problématiques, les professionnelles et professionnels apprécient le fait que leur travail leur offre une grande latitude qui leur permet de faire preuve de créativité et d'initiative.

Les professionnelles et professionnels rencontrés mentionnent comment les contacts humains et la solidarité sont très présents dans cette " communauté tissée serrée ", où tout 
le monde s'entraide. Il en est de même dans le milieu professionnel, où il est possible de contacter directement les supérieurs. Cependant, bien qu'il puisse paraitre avantageux d'être à la fois des amis et des collègues, cela présente aussi des défis lorsqu'il faut consulter une professionnelle ou un professionnel au sujet de problématiques personnelles. Il devient donc difficile de composer avec les frontières entre la vie privée et la vie professionnelle.

Parmi les participantes et participants du milieu professionnel francophone, certains déplorent l'absence de services de counseling en français. L'inaccessibilité à des services en français en santé mentale crée un sentiment de vulnérabilité pour ceux qui sont affectés par l'isolement ou par les facteurs environnementaux comme les conditions climatiques. De plus, ces professionnelles et professionnels rapportent que, malgré cette qualité de vie que procure le fait d'habiter dans une petite région comme Iqaluit, leur travail est exigeant et s'effectue très souvent dans des conditions difficiles : ils sont confrontés quotidiennement aux conditions de vie des Inuits, aux traumas et aux problèmes complexes qui découlent de la colonisation. En effet, l'effritement de la culture et la reproduction de la violence de manière intergénérationnelle créent un désespoir palpable qui affecte toute la population.

Les professionnelles et professionnels de la santé travaillent avec une clientèle très diversifiée dont les besoins varient énormément. Il s'agit donc d'un contexte qui laisse peu de place à la possibilité de développer une expertise dans un domaine spécifique. Ceux-ci doivent donc posséder un large éventail de connaissances, être prêts à faire face à des situations imprévues et se fier à leur jugement professionnel. Toutefois, la variété des tâches à accomplir rend leur travail intéressant et stimulant.

Les professionnelles et professionnels interrogés rapportent que la rétention du personnel est une grande difficulté à Iqaluit. Les postes vacants demeurent longtemps non pourvus, et le recrutement est difficile. Ces défis ont une incidence à la fois sur l'efficacité des intervenantes et intervenants, le ralentissement de l'offre de services essentiels et la continuité dans le suivi des dossiers.

Bien que la possibilité de vivre dans une communauté francophone ne soit pas la principale raison de venir s'établir à Iqaluit, les professionnelles et professionnels rencontrés mentionnent que la vitalité et la fierté qui émanent de la communauté francophone contribuent à renforcer leurs valeurs culturelles et linguistiques en milieu professionnel et à leur donner un sens identitaire qui les amène à s'engager dans la promotion et la défense de la langue française en situation minoritaire. Ils soulignent que beaucoup d'efforts ont été faits - et continuent d'être déployés — par les membres de la communauté et les organismes francophones pour faire connaître la communauté auprès des gens à l'extérieur $\mathrm{du}$ territoire et pour offrir du soutien aux francophones qui décident de venir vivre à Iqaluit. 
"Les professionnelles et professionnels disent être en accord avec les efforts de revitalisation et de promotion de l'inuktitut, mais ils sentent tout de même que les francophones sont relégués au dernier rang..."

Même si la langue française est reconnue officiellement par le gouvernement territorial $\mathrm{du}$ Nunavut, dans les faits, la situation n'est pas aussi simple. Les professionnelles et professionnels disent être en accord avec les efforts de revitalisation et de promotion de l'inuktitut, mais ils sentent tout de même que les francophones sont relégués au dernier rang et qu’ils doivent continuellement faire valoir leurs droits et leurs besoins auprès des organismes et du gouvernement.

Dans ce contexte, les résidentes et résidents s'attendent à ce que les gens qui viennent vivre à Iqaluit s'engagent et s'impliquent au sein de cette communauté. À cet effet, les professionnelles et professionnels soulignent qu'il s'agit d'un critère important lors du recrutement et de la sélection des employées et employés ou des stagiaires. Il est en effet mal vu de venir travailler à Iqaluit pendant un certain temps pour faire un coup d'argent, puis de repartir en ne laissant rien en retour. De plus, l'implication sociale et la participation aux activités communautaires favorisent l'intégration à l'arrivée, mais deviennent aussi par la suite un mode de vie.

\section{Discussion}

En lien avec notre cadre conceptuel, nous constatons que les stagiaires décrivent dans leurs propos le vécu du choc culturel, les exigences de l'adaptation, les éléments à développer sur le plan de la compétence culturelle et l'étape de décentration liée à une prise de conscience des différences culturelles internalisées. De même, elles abordent précisément l'humilité culturelle à développer en lien avec le sentiment d'impuissance qui les habite, elles développent une vision critique de l'offre des services et apprivoisent les éléments de sécurité culturelle qu'elles apprennent durant leur supervision de stage pour mieux ajuster leurs pratiques aux réalités locales. Tout au long de leur stage, elles sont soucieuses de mieux comprendre le fonctionnement social des familles rencontrées et d'agir respectueusement en cohérence avec les croyances des personnes qui utilisent des services, et elles tentent de décoder les comportements afin de déconstruire le courant dominant. Elles se placent dans une perspective d'ouverture pour aller à la rencontre d'inégalités sociales qui expliquent différemment les problématiques rencontrées, s'exercent à bien communiquer et à collaborer dans le respect de l'autodétermination du peuple inuit et montrent une prise de conscience des effets tangibles de la colonisation. 
les stages requièrent la mise en place de conditions spécifiques pour quils deviennent une expérience positive..."

Nos résultats appuient aussi ceux d'autres recherches qui insistent sur le fait que les stages requièrent la mise en place de conditions spécifiques pour qu'ils deviennent une expé-
Killam et Carter, 2010; Jones, Neubrander et rience positive (Moosa et Schurr, 2011; Killam et Carter, 2010; Jones, Neubrander et Huff, 2012). L'accès à la réalisation d'un stage en région éloignée lors de la formation académique et clinique des étudiantes et étudiants implique de bien connaitre les besoins professionnels, les réalités du travail et les actualités linguistiques et culturelles des communautés concernées (Keahey, 2008).

\section{Défis rencontrés}

Comme l'organisation des stages professionnels pose fréquemment des défis (Killam et Carter, 2010), la mise en œuvre du présent projet a été complexe et parfois difficile. Par conséquent, nous souhaitons présenter les défis rencontrés, ainsi que certaines stratégies pour l'organisation de stages interculturels en région éloignée et arctique.

Il importe de rappeler le contexte de ce projet, c'est-à-dire l'intention du Réseau de santé en français au Nunavut (RÉSEFAN) d'accueillir des stagiaires pouvant offrir des services en français à la population francophone au Nunavut, afin de répondre en partie aux problèmes de recrutement et de rétention de professionnelles et professionnels bilingues. De ce fait, afin que le milieu puisse accueillir des stagiaires, il a fallu premièrement élaborer diverses ententes interministérielles et interinstitutionnelles. Dans le cas d'une institution postsecondaire, certaines ententes n'ont pu être actualisées à temps. Par conséquent, l'occasion de stage n'a pas concordé avec les moments où les stagiaires devaient commencer le stage professionnel, selon leur cheminement de programme. Ainsi, certains stages ont dû être annulés à la dernière minute.

À titre de stratégie de rétention reconnue, le milieu d'accueil professionnel privilégie les stagiaires en provenance du territoire. Par ailleurs, ce milieu ne peut accueillir qu'un nombre limité de stagiaires, ce qui est dû en partie au manque de disponibilité des superviseuses et superviseurs potentiels dans un milieu de travail très chargé. Un défi additionnel semble être celui de la concurrence entre les institutions postsecondaires voulant intégrer des stagiaires dans la communauté d'Iqaluit. De plus, le recrutement et la rétention de professionnelles et professionnels de la santé et des services sociaux étant une des retombées visées par cette recherche, nous constatons que la capacité de la communauté d'Iqaluit à accueillir des stagiaires et les grands besoins de la communauté posent de sérieux défis à l'atteinte de ces objectifs. Paradoxalement, répondre aux besoins 
des francophones de cette région implique de développer des compétences spécifiques nécessaires au travail en région éloignée et en situation interculturelle, ce qui, à long terme, pourrait favoriser le recrutement et la rétention des professionnelles et professionnels de la santé.

Dans le cas de l'orthophonie au Nunavut, le seul professionnel exerçant ce métier est anglophone. L'offre de services en français n'est donc possible que lorsque des stagiaires francophones s'y rendent pour effectuer un stage clinique. De plus, si ce professionnel est incapable de superviser la ou le stagiaire, il doit y avoir de la supervision à distance. Cette situation occasionne un travail additionnel pour la professeure responsable des stages, comme il est préférable que ce soit une personne francophone qui identifie les dossiers auprès des organismes afin de préparer le stage. Ensuite, puisque le superviseur est anglophone, la ou le stagiaire devra avoir la capacité de travailler avec beaucoup d'autonomie. Dans de telles situations, il serait ainsi préférable de privilégier une ou un stagiaire qui est en fin d'études et qui possède une expérience clinique plus avancée.

"...nos analyses ont permis de dégager la grande complexité des problématiques sociales et structurelles de la communauté d'Iqaluit, ainsi que l'effet des défis de l'infrastructure (pénurie de logements) et de la colonisation sur la communauté inuite." d'œuvre qualifiée dans la communauté d'Iqaluit.
Finalement, nos analyses ont permis de dégager la grande complexité des problématiques sociales et structurelles de la communauté d'Iqaluit, ainsi que l'effet des défis de l'infrastructure (pénurie de logements) et de la colonisation sur la communauté inuite. Ces facteurs doivent donc figurer dans les stratégies pour contrer la pénurie de main-

\section{Recommandations}

Voici quelques recommandations issues des propos des participantes et participants (stagiaires et professionnelles et professionnels) qui pourraient contribuer au développement de stratégies efficaces pour la planification et la rétention de nouveaux professionnels et professionnelles en région éloignée.

Les écrits scientifiques montrent comment les étudiantes et étudiants qui complètent un stage en région éloignée doivent faire face à de nombreux obstacles comme l'isolement social et géographique, les coûts reliés au déplacement et à l'hébergement, le manque de soutien et l'accès limité aux ressources cliniques (Bradley, Bourke et Cosgrave, 2017). Ces obstacles sont présents tant du point de vue professionnel que du point de vue 
personnel (Organisation mondiale de la Santé, 2010). Playford, Larson et Wheatland (2006) rapportent également que ce type de milieu de stage implique aussi la séparation d'avec la famille et les amis, la dislocation sociale et, dans certains cas, la perte d'un revenu d'emploi. Tous ces obstacles doivent ainsi être considérés dans l'organisation des stages professionnels en milieu éloigné ou arctique.

\section{Préparation au stage}

Dans les communautés où il y a possibilité d'établir des milieux de stage, une visite sur le terrain s'avère une stratégie importante. Cette visite permet à l'équipe de gestion des stages de se familiariser avec la réalité géographique, politique, linguistique et culturelle de la région. Ensuite, lorsqu'un stage s'avère possible, les exigences légales (contrat d'affiliation) et médicales (immunisations) du territoire et des différents milieux de stage sont les prochaines préoccupations, et ce sont des impératifs avant de procéder à la planification avec les organismes du terrain. Par ailleurs, il faut déterminer les besoins de l'organisme d'accueil et des personnes qui superviseront et accueilleront la ou le stagiaire afin d'offrir les suivis d'encadrement du stage. De plus, il importe d'identifier un réseau ou des personnes pouvant accueillir la ou le stagiaire dans la communauté afin de faciliter son intégration et son adaptation. Finalement, dans le cas des stages qui ont été organisés à Iqaluit par l'Université Laurentienne, le RÉSEFAN ainsi que le Consortium national de formation en santé (CNFS) ont été des partenaires indispensables afin d'assurer leur succès.

\section{Préparation linguistique et culturelle}

Au-delà des détails logistiques pour les stagiaires, une préparation aux particularités linguistiques, culturelles et sociales du milieu de stage est essentielle, et ce, dans une perspective anti-oppressive. Il importe que les stagiaires soient sensibilisés aux divers contextes socioculturels et iniquités sociales afin de pouvoir agir dans le milieu en adoptant une posture qui privilégie la sécurité culturelle. Cette sensibilisation peut se faire par l'entremise de lectures et de discussions avec des personnes ayant complété un stage antérieur ou ayant visité la région. En effet, les barrières culturelles et linguistiques peuvent affecter l'atteinte des buts visés dans les plans préparatoires d'intervention (Moosa et Schurr, 2011; Killam et Carter, 2010). Comme les stagiaires plongeront dans la culture et la découverte, ils doivent aussi être préparés et prêts à l'adaptation culturelle, avoir une ouverture d'esprit quant aux différences linguistiques et culturelles, et être conscients des effets de l'oppression et de la colonisation. 


\section{Environnement et logement}

L'environnement et la préparation des stagiaires en lien avec les conditions climatiques et l'environnement sont aussi importants. Par conséquent, il importe au préalable de considérer la saison durant laquelle se feront les stages, les déplacements requis sur le terrain, ainsi que l'hébergement. Dans certaines communautés, la disponibilité de logement à court terme est parfois très difficile et complexe à organiser; il faut donc aller sur le terrain préalablement afin de déterminer un endroit approprié et sécuritaire pour les stagiaires. Enfin, étant donné que le coût de la vie est très élevé à Iqaluit (logement, nourriture, déplacement dans les diverses communautés), les stagiaires auront besoin d'un soutien financier additionnel.

\section{Particularités d'une région isolée}

Compte tenu de la nature du territoire (arctique, isolé et éloigné), les stagiaires doivent se préparer au contexte et aux différences rencontrées pour la pratique professionnelle. Certains milieux cliniques sont très exigeants et amènent les stagiaires à composer avec de nouvelles problématiques qu'ils n'ont possiblement pas approfondies durant leur formation, sans avoir toujours l'espace pour faire les recherches requises. De plus, les stagiaires doivent prendre en considération qu'il leur sera difficile de maintenir une vie privée au sein de ces communautés, ce qui constitue un facteur de stress supplémentaire, d'autant plus qu'ils seront loin de leur soutien social habituel. Il faut donc les préparer à faire face à ce type de situations et déterminer à l'avance des stratégies leur permettant de composer avec celles-ci. Il importe aussi d'ajuster les attentes, en favorisant la prise de conscience des petits gains, tout en étant conscient de ses limites.

\section{Intégration à la communauté}

Enfin, les stagiaires rapportent que le succès de l'expérience de stage dépend, en partie, de leur intégration dans la communauté durant toute sa durée. Il est recommandé aux stagiaires d'avoir une réelle volonté de contribuer à la communauté durant leur séjour dans le milieu et pendant tout le stage.

\section{Conclusion}

Ce projet de recherche est issu de l'intérêt que nous avons de mieux comprendre la portée des programmes de formation en santé et en santé mentale dans un contexte 
francophone minoritaire. Il a permis de documenter quelques-uns des nombreux défis liés à l'organisation des stages et de mieux comprendre les enjeux dans l'élaboration de nouveaux partenariats en milieu nordique et arctique. Cette étude empirique avait pour but aussi comme retombée sociale de contribuer à enrichir le potentiel des services en français pour la communauté francophone d'Iqaluit. En effet, l'établissement d'ententes de stages dans la région d'Iqaluit durant la formation professionnelle est une stratégie

"...ces stages influencent directement l'accès aux services de santé et services sociaux en français pour la population francophone d'Iqaluit. " efficace et reconnue pour faciliter le recrutement des professionnelles et professionnels de la santé (Fisher et Fraser, 2010). D’ailleurs, ces stages influencent directement l'accès aux services de santé et services sociaux en français pour la population francophone d'Iqaluit.

L'analyse des entretiens réalisés tend à montrer que les stages à Iqaluit représentent une occasion unique d'apprentissage pour les stagiaires. La diversité de la clientèle et l'étendue des besoins mènent les stagiaires non seulement à faire une synthèse des connaissances acquises durant leur formation universitaire, mais aussi à puiser dans leurs connaissances théoriques et pratiques afin de faire face à des situations imprévues et diversifiées. Malgré les défis liés à l'isolement et au choc culturel, cette expérience de stage au sein d'une autre communauté francophone en milieu minoritaire permet de consolider l'identité francophone des stagiaires et de bâtir leur confiance professionnelle.

Approfondir la connaissance de la réalité d'une communauté permet de mieux appréhender les multiples aspects complexes à prendre en considération dans l'organisation et le déroulement des stages et pour assurer le recrutement de professionnelles et professionnels qui souhaitent s'installer en région isolée, nordique ou arctique. Or, les entretiens avec les stagiaires ont permis de dégager les défis et les avantages de l'accomplissement d'un stage en milieu arctique. Par ailleurs, les propos des professionnelles et professionnels, quant à eux, ont offert un portrait de la communauté francophone afin de mieux comprendre les lacunes liées à l'organisation et à l'accès aux services en français. Si l'on combine les propos des stagiaires et des professionnelles et professionnels, on constate alors toute la complexité des besoins de cette communauté francophone qui côtoie la population inuite aux prises avec les effets de la colonisation. Dans cette réalité, il importe de reconnaître que les services en français ne peuvent être séparés du contexte géographique, historique, culturel et sociopolitique du territoire du Nunavut. 


\section{Notes}

1 Cette recherche a été rendue possible grâce à l'appui financier du Consortium national de formation en santé (CNFS), qui est financé par Santé Canada dans le cadre de la Feuille de route pour les langues officielles du Canada 2013-2018.

2 Bien que le gouvernement du Nunavut conserve l'invariabilité du nom et de l'adjectif « inuit ", nous avons choisi, par souci d'uniformisation, de suivre les recommandations du Bureau de la traduction du Canada ainsi que de l'Office québécois de la langue française et d'accorder, en genre et en nombre, l'adjectif ainsi que le nom « inuit ».

3 Personnes formant la communauté habitant le Nunavut.

\section{Bibliographie}

BÉLANGER, Mario (2002). "L'intervention interculturelle : une recherche de sens et un travail du sens ", Service social, Vol. 49, № 1, p. 70-93.

BLANCHET GARNEAU, Amélie, et Jacinthe PEPIN (2012). "La sécurité culturelle : une analyse du concept ", Recherche en soins infirmiers, No 111, p. 22-35.

BOUCHARD, Louise, et collab. (2009). «La santé en situation linguistique minoritaire », Healthcare Policy, Vol. 4, № 4, p. 36-42, réf. du 14 mars 2016, http://www.ncbi.nlm.nih.gov/pmc/articles

BOUCHARD, Louise, et collab. (2006). "Capital social, santé et minorités francophones ", Revue canadienne de santé publique, Volume 97, supplément 2, p. 17-21.

BRADLEY, Donna, Lisa BOURKE et Cath COSGRAVE (2017). Experiences of nursing and allied health students undertaking a rural placement - A study of barriers and enablers, [thèse de maîtrise], Université de Melbourne, réf. du 8 février 2017,

http://goingruralhealth.com.au/wp-content/uploads/Final-report-experiences-of-nursing-andallied-health-students-undertaking-a-rural-placement.pdf

BUREAU DU COMMISSAIRE AUX LANGUES DU NUNAVUT (2006). Loi sur les langues officielles du Nunavut, réf. du 30 avril 2018, http://langcom.nu.ca/sites/langcom.nu.ca/files/consLNun2008c17.pdf

BUTTON, Lori, et collab. (2005). «The impact of international placements on nurses' personal and professional lives: literature review ", Journal of Advanced Nursing, Vol. 50, p. 315-324.

CALLISTER, Lynn Clark, et Amy Harmer COX (2006). "Opening our hearts and minds: The meaning of international clinical nursing electives in the personal and professional lives of nurses ", Nursing and Health Sciences, Vol. 8, p. 95-102.

CAMPINHA-BACOTE, Josepha (2002). "The Process of Cultural Competence in the Delivery of Healthcare Services: A model of Care », Journal of Transcultural Nursing, Vol. 13, №3, p. 181-184. 
CARONNA, Carol A. (2010). "Why Use Qualitative Methods to Study Health Care Organizations? Insights from Multi-Level Case Studies ", dans Ivy Bourgeault, Robert Dingwall et Raymond De Vries (dirs.), The SAGE Handbook of Qualitative Methods in Health Research, Washington DC, SAGE, p. 71-87.

COHEN-EMERIQUE, Margalit (2011). Pour une approche interculturelle en travail social : théories et pratiques, Rennes, Presses de l'EHESP, $435 \mathrm{p}$.

COHEN-EMERIQUE, Margalit (1984). "Choc culturel et relations interculturelles de la pratique des travailleurs sociaux, formation à la méthode des incidents critiques ", Cahiers de sociologie économique et culturelle, № 7, p. 185-218.

CONSORTIUM NATIONAL DE FORMATION EN SANTÉ (CNFS) (2015). Documenter la complexité de l'offre de stages de formation dans les institutions de formation postsecondaire francophones et bilingues dans les CLOSM, [document de discussion], séance de travail du CNFS, le 3 juin 2015.

CRAGO, Martha, Shanley ALLEN et Wendy HOUGH-EYAMIE (1997). "Exploring innateness through cultural and linguistic variation: An Inuit example », dans M. Gopnik (dir.), The biological basis of language, Oxford, Oxford University Press, p. 70-90.

DENCH, Catherine, et collab. (2011). "The Development of an Inuktitut and English Language Screening Tool in Nunavut ", Canadian Journal of Speech Language Pathology and Audiology, Vol. 35, No 2, p. 168-76.

DOLBEC, André (2003). "La recherche-action ", dans Benoît Gauthier (dir.), Recherche sociale, Québec, Presses de l'Université du Québec, p. 505-540.

EVANSON, Tracy, et Barbara ZUST (2004). "The meaning of participation in an international service experience among baccalaureate nursing students ", International Journal of Nursing Education Scholarship, Vol. 1, p. 1-14.

FÉDÉRATION DES COMMUNAUTÉS FRANCOPHONES ET ACADIENNE DU CANADA (FCFA) (2006). Profil de la communauté francophone du Nunavut, Ottawa, FCFA, réf. du 20 novembre 2017, www.fcfa.ca/profils

FISHER, Karin, et John FRASER (2010). "Rural Health Career Pathways: Research Themes in Recruitment and Retention ", Australian Health Review, Vol. 34, p. 292-296.

FORGUES, Éric, et Rodrigue LANDRY (2014). L'accès aux services de santé en français et leur utilisation en contexte francophone minoritaire : rapport final, Institut canadien de recherche sur les minorités linguistiques, Moncton, Nouveau-Brunswick.

JONES, Anne-Marie, Judy NEUBRANDER et Marie HUFF (2012). «Global Partners in Education Journal ", Vol. 2, No 1, p. 1-11.

KEAHEY, Sheri (2008). "Against the Odds: Orienting and Retaining Rural Nurses ", Journal for Nurses in Staff Development, Vol. 24, № 2, p.15-20. 
KILLAM, Laura Anne, et Lorraine Mary CARTER (2010). "Challenges to the student nurse on clinical placement in the rural setting: a review of the literature ", Rural and Remote Health, Vol. 10, No 1523, p. 1-14, réf. du 20 novembre 2017, http://www.rrh.org.au

LECLERC, Jacques (2015). « Nunavut (Canada) », L’aménagement linguistique dans le monde, Québec, CEFAN, Université Laval, $1^{\text {er }}$ décembre 2015, réf. du 8 février 2018, http://www.axl.cefan.ulaval.ca/amnord/nunavut.htm

MERCURE, Dominique, Halimatou BA et Pierre TURCOTTE (2010). "La formation pratique lors d'un stage international en travail social et le développement d'habiletés en intervention interculturelle ", Revue Intervention, Vol. 132, № 1, p. 44-52.

MILES, Matthew, Michael HUBERMAN et Johnny SALDAÑA (2014). Qualitative Data Analysis. A Methods Sourcebook, Thousand Oaks, SAGE.

MOOSA, Taslim, et Susan SCHURR (2011). "Reflections on a Northern Ontario Placement Initiative ", Canadian Journal of Speech-Language Pathology and Audiology, Vol. 35, № 2, p. 160-167.

ORGANISATION MONDIALE DE LA SANTÉ (2010). Increasing access to health workers in remote and rural areas through improved retention, Global Policy Recommendations, réf. du 8 février 2017, http://www.who.int/hrh/retention/guidelines/en/

PLAYFORD, Denese, Ann LARSON et Belynda WHEATLAND (2006). "Going country: Rural student placement factors associated with future rural employment in nursing and allied health ", Australian Journal of Rural Health, Vol. 14, p. 14-19, réf. du 3 octobre, 2017, doi: 10.1111/j.14401584.2006.00745.x

RIDDICK, Sherry (1998). "Improving Access for Limited English-Speaking Consumers: A Review of Strategies in Health Care Settings ", Journal of Health Care for the Poor and Underserved, Vol. 9, p. S40-S61.

SALDAÑA, Johnny (2013). The Coding Manual for Qualitative Researchers, Thousand Oaks, SAGE.

STATISTIQUE CANADA (2016). Recensement de 2016, réf du $1^{\mathrm{er}}$ avril 2018, http://www12.statcan.gc.ca/census-recensement/2016/dp-pd/prof/details/page.cfm?Lang=F\&G eo $1=$ CD\&Code1 $=6204 \&$ Geo2 $=$ PR \&Code2 $=62 \&$ Data $=$ Count $\&$ SearchText $=$ Baffin $\&$ Search Ty pe $=$ Begins $\&$ SearchPR $=01 \& B 1=$ All $\&$ GeoLevel $=P R \&$ GeoCode $=6204 \&$ TABID $=1$

STATISTIQUE CANADA (2012). GéoRecherche, Recensement de 2011, № 92-142-XWF au catalogue de Statistique Canada, Données mises à jour le 24 octobre 2012, Ottawa, Statistique Canada.

WALSH, Linda, et Jeanne DEJOSEPH (2003). « I saw it in a different light: International learning experiences in baccalaureate nursing education ", Journal of Nursing Education, Vol. 42, p. 266-272. 